\title{
A SHORT NOTE ON FIBONACCI NUMBERS IN A GENERALIZED PYTHAGOREAN TRIPLES
}

\author{
Emily L. Casinillo ${ }^{1}$ and Leomarich F. Casinillo ${ }^{2 *}$ \\ ${ }^{1,2}$ Department of Mathematics and Physics \\ Visayas State University, Visca, Baybay City, Leyte, Philippines \\ Email : ${ }^{2}$ leomarichcasinillo02011990@gmail.com \\ "Penulis Korespondensi
}

\begin{abstract}
This paper aims to construct a new formula that generates a Fibonacci numbers in a generalized Pythagorean triples. In addition, the paper formulates some Fibonacci identities and discuss some important findings.
\end{abstract}

Keywords: Fibonacci numbers, Pythagorean triples, Fibonacci identities

\section{PENDAHULUAN}

Through various years, Fibonacci's famous number sequence has been a source of a lot of intriguing mathematical investigations and researches [14]. Fibonacci sequence is one of the interesting topics in combinatorics and number theory that intrigued and inspired a lot of mathematicians for many years $[1,2,7,9,16]$. A Fibonacci number $F_{n}$ is defined recursively as $F_{n}=F_{n-1}+F_{n-2}$ for $n \geq 2$, and $F_{0}=0, F_{1}=1[3,4]$. The concept of Fibonacci numbers has a lot of interesting applications in physical sciences $[8,18]$. Some applications of Fibonacci numbers can also be found in the area of graph theory [4, 5, 15, 17]. In addition, several mathematicians are inspired of dealing Fibonacci identities that can be proven by induction, generating functions, determinants and so on $[2,3,14,16]$. On the other hand, a Pythagorean triple $(X, Y, Z)$ is a triple of positive integers such that $X^{2}+Y^{2}=Z^{2}$. A Pythagorean triple is said to be primitive if the greatest common divisor of the triple is 1 , and each pair of integers $X, Y$, and $Z$ are relatively prime, otherwise the triple is known as non-primitive $[6,7,9]$. Pythagorean triple is considered as one of the oldest topic in number theory that remains intriguing and receiving much attention to research $[6,7,9,10,11]$.

In the paper of Casinillo and Casinillo [6], an alternative formula that generates Phytagorean triples was construted. And this formula is called the generalized version of Pythagorean triples since it generates a primitive and non-primitive triples. However, the formula does not generates all Pythagorean triples. It is worthy to note that some Pythagorean triples are scalar multiples of other triples, hence, to generate all triples, one may consider a scalar positive integer to the generalized version of Pythagorean triples. Another paper of Casinillo and Casinillo [7] generates a new formula for generalized version of congruent numbers based on a generalized version of Pythagorean triples. Furthermore, an elliptic curve equation was also constructed from generalized version of Pythagorean triples. Several mathematicians studied the deep connection of Fibonacci numbers and Pythagorean triples which resulted to numerous papers in literature $[10,12,13,14]$. Hence, the purpose of this paper is to construct a new formula that generates a Fibonacci numbers in a generalized version of Pythagorean triples. In addition, the paper formulates some Fibonacci identities and prove using mathematical induction. 


\section{RESULTS}

The following theorem shows that a Pythagorean triple $(X, Y, Z)$ is a function of two distinct Fibonacci numbers, that is, $X=\delta_{1}\left(F_{n}, F_{m}\right), Y=\delta_{2}\left(F_{n}, F_{m}\right)$, and $Z=\delta_{3}\left(F_{n}, F_{m}\right)$, where $n$ and $m$ are positive integers and $\delta_{i}:\left(\mathbb{Z}^{+}\right)^{2} \rightarrow \mathbb{Z}^{+}$for $i \in\{1,2,3\}$. The proof of the theorem is based on the current study by Casinillo and Casinillo [6] which deals with generalized Pythagorean triples. It is worthy to note that Theorem 1 also generates primitive and non-primitive Pythagerean triples.

Theorem 1. Let $F_{n}$ and $F_{m}$ be Fibonacci numbers where where $n$ and $m$ are positive integers. If $(X, Y, Z)$ is a Pythagorean triple, then $X=2 F_{n}^{2}+2 F_{m} F_{n}, Y=2 F_{m} F_{n}+F_{m}^{2}$, and $Z=2 F_{n}^{2}+$ $2 F_{m} F_{n}+F_{m}^{2}$

Proof. Suppose that $(X, Y, Z)$ is a Pythagorean triple. Then, it satisfies the Diophantine equation

$$
X^{2}+Y^{2}=Z^{2}
$$

So, by algebra, it follows that

$$
\frac{(Z+Y)(Z-Y)}{X^{2}}=1
$$

Rewritting the equation (2), we obtain

$$
\left(\frac{Z+Y}{X}\right)\left(\frac{Z-Y}{X}\right)=1=\left(\frac{Z}{X}+\frac{Y}{X}\right)\left(\frac{Z}{X}-\frac{Y}{X}\right)
$$

We suppose that $F_{n}$ and $F_{m}$ be Fibonacci numbers. Now, we let

$$
\frac{Z}{X}+\frac{Y}{X}=\frac{F_{n}+F_{m}}{F_{n}}
$$

and

$$
\frac{Z}{X}-\frac{Y}{X}=\frac{F_{n}}{F_{n}+F_{m}}
$$

By algebra, we can manipulate equation (4) and (5) and arrive the following equations

and

$$
\frac{Z}{X}=\frac{2 F_{n}^{2}+2 F_{m} F_{n}+F_{m}^{2}}{2 F_{n}^{2}+2 F_{m} F_{n}}
$$

$$
\frac{Y}{X}=\frac{2 F_{m} F_{n}+F_{m}^{2}}{2 F_{n}^{2}+2 F_{m} F_{n}}
$$

Hence, it is clear that

$$
(X, Y, Z)=\left(2 F_{n}^{2}+2 F_{m} F_{n}, 2 F_{m} F_{n}+F_{m}^{2}, 2 F_{n}^{2}+2 F_{m} F_{n}+F_{m}^{2}\right)
$$

This completes the proof.

The corollary and remarks below are direct consequence of Theorem 1 .

Corollary 2. Let $\left(X\left(F_{n}, F_{m}\right), Y\left(F_{n}, F_{m}\right), Z\left(F_{n}, F_{m}\right)\right)$ be a Pythagorean triple where $F_{n}$ and $F_{m}$ are Fibonacci numbers. If $N$ is a congruent number, then $N=2 F_{m} F_{n}^{3}+3 F_{m}^{2} F_{n}^{2}+F_{m}^{3} F_{n}$.

Proof. Immediate from Theorem 1.

Remark 3. Let $F_{n}$ and $F_{m}$ are Fibonacci numbers. Then, the following holds:

i. $\quad Z\left(F_{n}, F_{m}\right)>Y\left(F_{n}, F_{m}\right)>X\left(F_{n}, F_{m}\right)>6$ where $n<m$; and

ii. $\quad Z\left(F_{n}, F_{m}\right)>X\left(F_{n}, F_{m}\right)>Y\left(F_{n}, F_{m}\right) \geq 3$ where $n \geq m$. 
Remark 4. Let $\left(X\left(F_{n}, F_{m}\right), Y\left(F_{n}, F_{m}\right), Z\left(F_{n}, F_{m}\right)\right)$ be a Pythagorean triple where $F_{n}$ and $F_{m}$ are Fibonacci numbers. If $\cos ^{2}\left[\pi \frac{(Z-1) !+1}{Z}\right]=1$, then $Z$ is a Pythagorean prime.

The following lemma below is useful in proving our next new results. For the sake of curiosity, we provide alternative (detailed) proofs using mathematical induction to the following interesting Fibonacci identities showing that it is true for all positive integers.

Lemma 5. [2, 3] Let $F_{n}$ be a Fibonacci number and $n$ is a positive integer. Then, the following identities holds:

$$
\begin{array}{ll}
\text { i. } & F_{2 n+1}=F_{n+1}^{2}+F_{n}^{2} ; \text { and } \\
\text { ii. } & F_{2 n}=F_{n}\left(F_{n+1}+F_{n-1}\right) .
\end{array}
$$

Proof. Case (i). We let $A(n)$ is the statement $F_{2 n+1}=F_{n+1}^{2}+F_{n}^{2}$ where $n$ is a positive integer. Now, we consider the base case $A(1)$ as follows

$$
\begin{gathered}
F_{2(1)+1}=F_{1+1}^{2}+F_{1}^{2} \\
F_{3}=F_{2}^{2}+F_{1}^{2} \\
2=2
\end{gathered}
$$

Hence, $A(1)$ is true. In this case, our induction hypothesis is the statement $A(n)$. Then, we show that $A(n+1)$ is true, that is, $F_{2(n+1)+1}=F_{2 n+3}$. By definition of Fibonacci numbers, we obtain $F_{2(n+1)+1}=F_{2 n+2}+F_{2 n+1}=F_{2(n+1)}+F_{2 n+1}$. So, from our assumption hypothesis, we get $F_{2(n+1)+1}=F_{n+1}\left(F_{n+2}+F_{n}\right)+F_{n+1}^{2}+F_{n}^{2}$. Again, by definition of Fibonacci numbers and by simple algebra, we have

$$
\begin{aligned}
F_{2(n+1)+1} & =\left(F_{n+1}^{2}+2 F_{n} F_{n+1}+F_{n}^{2}\right)+F_{n+1}^{2} \\
& =\left(F_{n+1}+F_{n}\right)^{2}+F_{n+1}^{2} \\
F_{2(n+1)+1}= & F_{n+1}^{2}+F_{n}^{2}
\end{aligned}
$$

Thus, it is proven that $A(n+1)$ is true.

Case (ii). Again, we let $B(n)$ be the statement $F_{2 n}=F_{n}\left(F_{n+1}+F_{n-1}\right)$ where $n$ is a positive integer. First, we consider the base case $B(1)$ as follows:

$$
\begin{aligned}
& F_{2(1)}=F_{1}\left(F_{1+1}+F_{1-1}\right) \\
& F_{2}=F_{1}\left(F_{2}+F_{0}\right) \\
& 1=1
\end{aligned}
$$

This implies that $B(1)$ holds true. Assuming that our induction hypothesis is the statement $B(n)$. Secondly, we want to show that $B(n+1)$ is also true. So, we get $F_{2(n+1)}=F_{2 n+2}$. Then, by definition of Fibonacci numbers, we have $F_{2(n+1)}=F_{2 n+1}+F_{2 n}$. Hence, from the induction hypothesis, we obtain $F_{2(n+1)}=F_{n+1}^{2}+F_{n}^{2}+F_{n}\left(F_{n+1}+F_{n-1}\right)$. By algebra and by definition of Fibonacci number, we have the following

$$
\begin{aligned}
F_{2(n+1)} & =F_{n+1}\left(F_{n}+F_{n+1}\right)+F_{n}\left(F_{n}+F_{n-1}\right) \\
& =F_{n+1} F_{n+2}+F_{n} F_{n+1} \\
F_{2(n+1)} & =F_{n+1}\left(F_{n+2}+F_{n}\right)
\end{aligned}
$$

This shows that $B(n+1)$ is true for all positive integer $n$. Combining the two cases completes the proof. 
The next lemma below is a direct consequence of Lemma 5 showing that the Pythagorean triple $(X, Y, Z)$ is a function of Fibonacci number.

Lemma 6. [11] Let $(X, Y, Z)$ be Pythagorean triple. If $n \geq 2$, then

$$
\left(X=2 F_{n} F_{n+1}, Y=F_{n+1}^{2}-F_{n}^{2}, Z=F_{2 n+1}\right)
$$

Proof. Follows directly from Lemma 5.

Our next result is quick from Theorem 1, Lemma 5 and Lemma 6 above. This shows a new interesting Fibonacci identities which can be proven by induction.

Theorem 7. Let $k \geq 2$. If $(X, Y, Z)$ is a Pythagorean triple, then

$$
\begin{array}{ll}
\text { i. } & X=2 F_{k} F_{k+1}=2 F_{k}^{2}+2 F_{k-1} F_{k} ; \\
\text { ii. } & Y=F_{k+1}^{2}-F_{k}^{2}=F_{k-1}^{2}+2 F_{k-1} F_{k} ; \text { and } \\
\text { iii. } & Z=F_{2 k+1}=2 F_{k}^{2}+2 F_{k-1} F_{k}+F_{k-1}^{2} .
\end{array}
$$

Proof. Let $k \geq 2$. Then, by Theorem 1 , it is easy to check that

$$
(X, Y, Z)=\left(2 F_{k}^{2}+2 F_{k-1} F_{k}, F_{k-1}^{2}+2 F_{k-1} F_{k}, 2 F_{k}^{2}+2 F_{k-1} F_{k}+F_{k-1}^{2}\right)
$$

for $n=k=m+1$. Now, we consider the following 3 cases below:

Case $(i)$. We let $C(k)$ is the statement $2 F_{k} F_{k+1}=2 F_{k}^{2}+2 F_{k-1} F_{k}$ where $k$ is a positive integer greater than 1 . Now, we consider the base case $C(2)$ as follows

$$
\begin{gathered}
2 F_{2} F_{2+1}=2 F_{2}^{2}+2 F_{2-1} F_{2} \\
2 F_{2} F_{3}=2 F_{2}^{2}+2 F_{1} F_{2} \\
2(1)(2)=2(1)^{2}+2(1)(1) \\
4=4
\end{gathered}
$$

Hence, the base case $C(2)$ is true. Our induction hypothesis is the statement $C(k)$. So, we will show that $C(k+1)$ holds. By definition of Fibonacci numbers, we obtain

Hence, it is proven that $C(k+1)$ is true.

$$
\begin{aligned}
2 F_{k+1} F_{k+2} & =2 F_{k+1}\left(F_{k}+F_{k+1}\right) \\
& =2 F_{k+1}^{2}+2 F_{k} F_{k+1}
\end{aligned}
$$

Case (ii). Again, let $D(k)$ be the statement $F_{k+1}^{2}-F_{k}^{2}=F_{k-1}^{2}+2 F_{k-1} F_{k}$ where $k$ is a positive integer greater than 1 . So, consider the base case $D(2)$, we have

$$
\begin{aligned}
F_{2+1}^{2}-F_{2}^{2} & =F_{2-1}^{2}+2 F_{2-1} F_{2} \\
F_{3}^{2}-F_{2}^{2} & =F_{1}^{2}+2 F_{1} F_{2} \\
(2)^{2}-(1)^{2} & =(1)^{2}+2(1)(1) \\
3 & =3
\end{aligned}
$$

This implies that $D(2)$ holds true. Assume that the induction hypothesis is the statement $D(k)$. Hence, we want to show that $D(k+1)$ is also true. By definition of Fibonacci numbers, we obtain

$$
\begin{aligned}
& F_{k+2}^{2}-F_{k+1}^{2}=\left(F_{k}+F_{k+1}\right)^{2}-\left(F_{k-1}+F_{k}\right)^{2} \\
& \quad=F_{k}^{2}+2 F_{k} F_{k+1}+F_{k+1}^{2}-F_{k-1}^{2}-2 F_{k-1} F_{k}+F_{k}^{2}
\end{aligned}
$$

By simplifying equation (33) and from our assumption, we get the following

$$
\begin{gathered}
F_{k+2}^{2}-F_{k+1}^{2}=2 F_{k} F_{k+1}+F_{k+1}^{2}-F_{k+1}^{2}+F_{k}^{2}+2 F_{k-1} F_{k}-2 F_{k-1} F_{k} \\
F_{k+2}^{2}-F_{k+1}^{2}=F_{k}^{2}+2 F_{k} F_{k+1}
\end{gathered}
$$

This shows that $D(k+1)$ also holds.

Case (iii). Lastly, we let $E(k)$ be the statement $F_{2 k+1}=2 F_{k}^{2}+2 F_{k-1} F_{k}+F_{k-1}^{2}$ where $k$ is a positive integer greater than 1 . Consider the base case $E(2)$, so we have 


$$
\begin{aligned}
F_{2 k+1} & =2 F_{k}^{2}+2 F_{k-1} F_{k}+F_{k-1}^{2} \\
F_{2(2)+1} & =2 F_{2}^{2}+2 F_{2-1} F_{2}+F_{2-1}^{2} \\
F_{5} & =2 F_{2}^{2}+2 F_{1} F_{2}+F_{1}^{2} \\
5 & =5
\end{aligned}
$$

It follows that $E(2)$ holds. Next, we assume that the induction hypothesis is the statement $E(k)$. So, we want to show that $E(k+1)$ holds true. Hence, we have

In view of Lemma 5 , we obtain

$$
\begin{aligned}
F_{2(k+1)+1} & =F_{2 k+3} \\
& =F_{2 k+2}+F_{2 k+1} \\
& =F_{2(k+1)}+F_{2 k+1}
\end{aligned}
$$

$$
F_{2(k+1)+1}=F_{k+1}\left(F_{k+2}+F_{k}\right)+F_{k+1}^{2}+F_{k}^{2}
$$

By definition of Fibonacci number and simplifying equation (43), we end up with

$$
F_{2(k+1)+1}=2 F_{k+1}^{2}+2 F_{k} F_{k+1}+F_{k}^{2}
$$

This implies that $E(k+1)$ is true. Hence, combining the 3 cases, completes that proof.

The following corollary below shows a new Fibonacci identity for the hypothenuse of a right triangle. This result is quick from Theorem 1 and Lemma 6.

Corollary 8. Let $(X, Y, Z)$ be a Pythagorean triple. For any $k \geq 2$, we have

$$
Z=F_{k+1}^{2}+F_{k}^{2}=2 F_{k}^{2}+2 F_{k-1} F_{k}+F_{k-1}^{2}
$$

Proof. Note that by Lemma 6 , we have $X=2 F_{k} F_{k+1}$ and $Y=F_{k+1}^{2}-F_{k}^{2}$. Solving $Z$ in the equation $X^{2}+Y^{2}=Z^{2}$, we obtain $Z=F_{k+1}^{2}+F_{k}^{2}$. By Theorem $1, Z=F_{2 k+1}=2 F_{k}^{2}+$ $2 F_{k-1} F_{k}+F_{k-1}^{2}$. Clearly, the hypothesis follows and this completes the proof.

\section{CONCLUSION}

In this paper, we had developed a new formula that generates a Fibonacci numbers in a generalized Pythagorean triples. Furthermore, the paper formulated some Fibonacci identities and proved using induction. For future research, one must investigate the connection of generalized version of congruent numbers [7] and Fibonacci numbers.

\section{CONCLUSION}

The authors are very grateful to the referees for the rigorous review and suggestions which led to the improvement of this paper.

\section{REFERENCES}

[1] Aires, A. P., Catarino, P., Campos, H., Borges, A., \& Vasco, P. (2014). s-Generalized Fibonacci numbers: some identities, a generating function and Pythagorean triples. Int. J. Math. Anal, 8(36), 1757-1766.

[2] Askey, R. A. (2004). Delving Deeper: Fibonacci and Related Sequences. The Mathematics Teacher, 97(2), 116-119.

[3] Benjamin, A. T. \& Quinn, J. J. (1999). Recounting Fibonacci and Lucas identities. The College Mathematics Journal, 30(5), 359-366. 
[4] Casinillo, L. F., "A note on Fibonacci and Lucas number of domination in path," Electronic Journal of Graph Theory and Applications, vol. 6, no. 2, pp. 317-325, 2018.

[5] Casinillo, L. F., "Odd and even repetition sequences of independent domination number," Notes on Number Theory and Discrete Mathematics, vol. 26, no. 1, pp. 8-20, 2020.

[6] Casinillo, L. F., \& Casinillo, E. L., "Some notes on a generalized version of Pythagorean triples," Jurnal Riset dan Aplikasi Matematika (JRAM), vol. 4. no. 2, pp. 103-107, 2020.

[7] Casinillo, L. F., \& Casinillo, E. L., "Some Results on a Generalized Version of Congruent Numbers", InPrime: Indonesian Journal of Pure and Applied Mathematics, vol. 3, no. 1, pp. 1-6, 2021.

[8] D'Amico, A., Faccio, M. and Ferri, G., "Ladder network characterization and Fibonacci numbers," Nuovo Cimento D, vol. 12, no. 1, pp. 1165-1173, 1990.

[9] Erickson, M. and Vazzana, A., Introduction to Number Theory. New York: Taylor and Francis Group, 2008.

[10] Gil, B. K., Han, J. W., Kim, T. H., Koo, R. H., Lee, B. W., Lee, J., ... \& Park, P. S., "Frobenius numbers of Pythagorean triples," International Journal of Number Theory, vol. 11, no. 02, pp. 613-619, 2015.

[11] Hammes, E., Unifications of Pythagorean Triple Schema, Undergraduate Honors Theses. Paper 502, 2019. https://dc.etsu.edu/ honors/502

[12] Horadam, A. F., "Fibonacci number triples," The American Mathematical Monthly, vol. 68, no. 8, pp. 751-753, 1961.

[13] Leyendekkers, J. V., \& Shannon, A. G., "Primitive Pythagorean triples and generalized Fibonacci sequences," Notes on Number Theory and Discrete Mathematics, vol. 23, no. 1, pp. 54-62. 2017.

[14] Pagni, D., "Fibonacci meets Pythagoras," Mathematics in School, vol. 30, no. 4, pp. 3942, 2001.

[15] Prodinger, H., \& Tichy, R., "Fibonacci numbers of graphs", The Fibonacci Quarterly, vol. 20, no. 1, pp. 16-21, 1982.

[16] Rabinowitz, S., Algorithmic manipulation of Fibonacci identities in Applications of Fibonacci numbers,. pp. 389-408, Dordrecht: Springer, 1996.

[17] Startek, M., Włoch, A., \& Włoch, I., "Fibonacci numbers and Lucas numbers in graphs," Discrete applied mathematics, vol. 157, no. 4, pp. 864-868, 2009.

[18] Tracy, C. A., "Universality class of a Fibonacci Ising model," Journal of Statistical Physics, vol. 51, no. 3-4, pp. 481-490, 1988. 